\title{
LUMINOSITY FUNCTION, STAR DENSITY, AND STAR FORMATION EFFICIENCY IN REGIONS OF STAR FORMATION
}

\author{
- NEAR INFRARED OBSERVATIONS - \\ Klaus-Werner Hodapp \\ John Rayner \\ Hua Chen \\ Institute for Astronomy, University of Hawaii \\ 2680 Woodlawn Drive, Honolulu, HI 96822, U.S.A.
}

\begin{abstract}
Clusters of young stars have been found near a number of compact HII regions. These clusters do not show a turnover in the K-band luminosity and are probably several million years old. In L 1641 only moderate clustering tendency has been observed and many sources show signs of extremely young age.
\end{abstract}

\section{Introduction}

Near infrared (NIR) observations, here meaning the wavelength range from $1.0-2.5 \mu \mathrm{m}$, can penetrate about an order of magnitude deeper into molecular clouds than visible light studies. In typical starforming molecular clouds, most embedded stars become visible in the NIR. The most heavily obscured very young stars escape direct detection even at $2.2 \mu \mathrm{m}$ and are often only seen in scattered light. Obtaining luminosity information requires extinction correction which however are still unreliable since the NIR extinction law is poorly known in molecular clouds. NIR detector technology has matured rapidly in the last 5 years. We can expect that in the near future, all the basic observational techniques of optical astronomy (imaging, photometry, polarimetry, and spectroscopy) will also be available in the NIR. This paper reports initial results of NIR imaging observations of starforming regions, obtained during the last 2 years at the University of Hawaii.

\section{S 106}

The first object we want to discuss is $S 106$, for which we have the most complete results. S 106 is a bipolar HII region excited by a 09 or B0 star. The bipolar morphology of S 106 in the radio continuum is commonly explained by an anisotropic stellar wind blocking UV photons from ionizing the equatorial plane of the bipolar structure (Felli et al. 1984). In addition, optical and infrared images as well as molecular line studies show that the object is surrounded by a clumpy torus of molecular gas, whose dust absorption leads to the broad absorption band bisecting the HII region at visible and NIR wavelengths.

Our $\mathrm{K}$ band image of this region is a mosaic of 129 frames obtained with a 128x128 HgCdTe detector array. The image covers a 4'x7' field of view. In the extended emission east of the illuminating star, some linear features are extending away from the central source into a region of high extinction in the dust lane. We interpret these features as illumination (shadow and light) effects of a clumpy molecular gas and dust distribution around the central star, being projected into the dense molecular material east of this star and becoming visible by scattering. 
Even considering the uncertainties in where exactly the clumpy material is located, this projection effect allows a "magnified" view of this clumpy material, confirming the existence of clumpiness on the scale of $1 "=600 \mathrm{AU}$ in the molecular torus around S 106.

Fig. 1: K-band mosaic image of S 106, obtained with a 128x128 HgCdTe infrared array.

Another but maybe more important feature in Fig. 1 is a cluster of approx. 200 stars surrounding the bipolar nebula. The center of this cluster is located some 30 " NW of the ionizing star, i.e. the latter is not located precisely in its center.

Quantitative photometric analysis of mosaic images like Fig. 1 is very difficult. Due to responsivity nonuniformities of the array, the noise is not constant in each flatfieldcorrected frame. Sky background and to some extent the PSF vary from frame to frame. In addition, source crowding and the extended nebulosity make photometry very difficult and automated source searches prone to errors. To check the reliability of our search routines under these circumstances, we inserted fake stars with the same PSF 'and noise properties than real images into the frame at random locations. The probability of rediscovering these fake stars by the search routine was then measured. Fake stars fainter than $\mathrm{K}=12$ show increasing photometric errors and at $\mathrm{K}=14$, the rediscovery rate drops rapidly. Down to this completeness limit of $K=14$, the $K$-band magnitude distribution rises smoothly. Beyond the completeness limit, it drops rapidly to zero, as expected. We do not see any indication for a turnover in this brightness distribution.

Only some very tentative results on the evolutionary status of the stars in the S 106 cluster can be derived from a K-band image alone. The majority of stars in this cluster are not associated with localized reflection nebulosity, that is frequently found around very young stars in their outflow phase. Only 4 stars, including IRS 4 show localized reflection nebulosity around them (as opposed to the generally distributed nebulosity in this region). Polarization studies of stars in the S 106 area have only found one star showing strong intrinsic polarization, which is also an indicator of young age (HL Tau being a prototype for such a strongly polarized young T Tauri star). Taken together, only 5 stars out of 200 
stars detected in total show signs of extreme youth associated with the formation of a dense disk and the outflow phase, commonly assumeed to last about 50000 years (Fukui, 1989). Assuming that the star formation rate in $S 106$ was constant in the past, this leads to a very rough estimate for the age of the oldest stars in $S 106$ of 2 million years. The assumption of constant star formation rate may of course be wrong and star formation may have proceeded in bursts, but the above estimate indicates that the $S 106$ cluster as a whole is not an extremely young structure, and that the individual stars in this cluster may have a substantial spread in their ages.

\section{Clusters associated with other HII regions}

S 106 is not a unique object. We are currently conducting a survey of a sample of compact HII regions in an attempt to study the frequency of cluster formation. We are concentrating on HII regions in the distance range from 2 to $5 \mathrm{Kpc}$, which can be imaged on a single frame of our infrared camera. In the majority of cases studied, we have found young embedded clusters, examples being S 255, S 269, W3(OH), and GL 437 (Rayner et al. 1990). The formation of groups or clusters of young stars therefore seems to be common in molecular clouds massive enough to form $\mathrm{O}$ or B stars sometimes during their star formation phase.

\section{The L 1641 Cloud}

In an attempt to study the star formation efficiency in a whole cloud, we obtained NIR images of outflow sources in L 1641. L 1641 is the southern part of Orion A and, following the data presented by Bally (this conference) and Fukui (this conference) the L 1641 cloud consists of two molecular filaments that are being twisted around each other, thereby creating a dense region where, according to the IRAS survey, most of the star formation occurs. The winding up of the filaments progresses along the axis of $\mathrm{L}$ 1641 from north to south. In addition, there is evidence that further to the north, the Orion A cloud has been compressed by the passage of a shock front. In summary, the available data suggest that star formation is spreading through the Orion A cloud from north to south.

NIR imaging of outflow sources in Orion A seems to confirm this result. In the north, there is the dense and rich cluster of young stars associated with the Trapezium and the BN/KL complex (McCaughrean, 1989). Using similar arguments as in the case of S 106, it can again be concluded that most stars in this cluster are not of extremely young age. Further to the south, the moderately populous cluster associated with the L $1641 \mathrm{~N}$ outflow source (Strom et al. 1989) (Fig.2 a) contains a larger number of objects associated with reflection nebulosity. Around some of the other outflow sources in L 1641 (Fig. 2 b, c, and d), still further south, the number of stars seen per unit area (to the same limiting magnitude) decreases, but the fraction of stars showing localized reflection nebulosity increases, as can be seen from the images in Fig. 2. We take this as evidence that in this southern part of L 1641, a large fraction of all stars visible are in fact very yqung and that star formation has been going on for only a relatively short time (a few $10^{5}$ years, to give a rough estimate). Clearly, the presence of reflection nebulosity is a poor indicator for the age of a star, but it is the only one available to date. Emission line spectroscopy of individual stars in a cluster will hopefully be a more powerful indicator of accreation disk activity and age. 

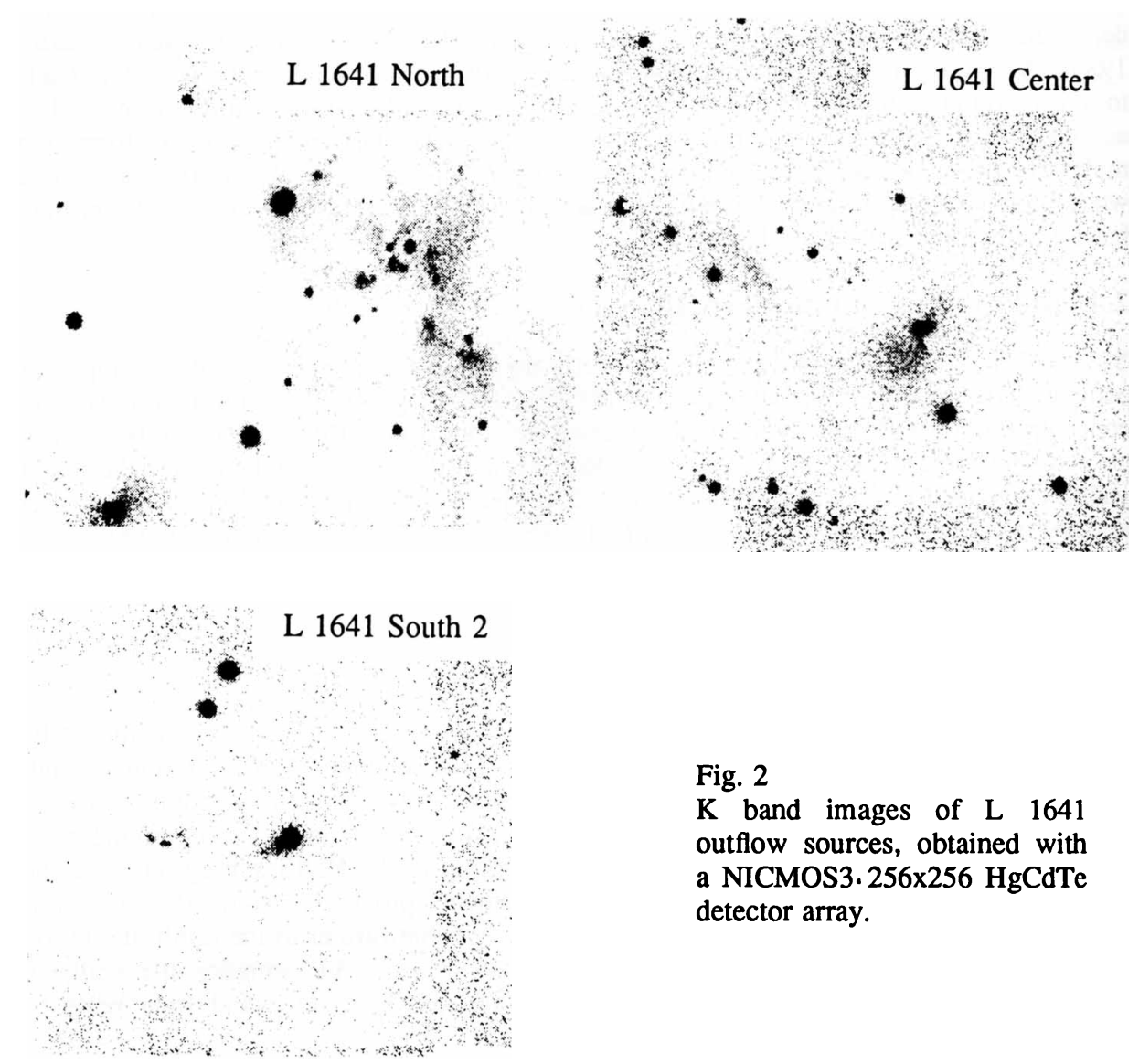

Fig. 2

$\mathrm{K}$ band images of $\mathrm{L} 1641$ outflow sources, obtained with a NICMOS3. $256 \times 256 \mathrm{HgCdTe}$ detector array.

\section{References}

Felli, M., Staude, H.J., Reddman, T., Massi, M., Eiroa, C., Hefele, H., Neckel, T., and Panagia, N., 1984, Astr. Ap, 135, 261.

Fukui, Y. 1989, ESO Workshop on Low Mass Star Formation and Pre-Main Sequence Objects, ed. B. Reipurth, ESO Conference and Workshop Proceedings No. 33, p. 447.

McCaughrean, M. 1989, Proceedings of the Third Infrared Detector Technology Workshop, ed. Craig R. McCreight, NASA Technical Memorandum 1C2209, p. 201.

Rayner, J., Hodapp, K.-W., Zinnecker, H. 1990, Astrophysics with Infrared Arrays, Tucson, proceedings in press.

Strom, K.M., Margulis, M., Strom, S.E. 1989, Ap. J. Letters, 346, L33. 